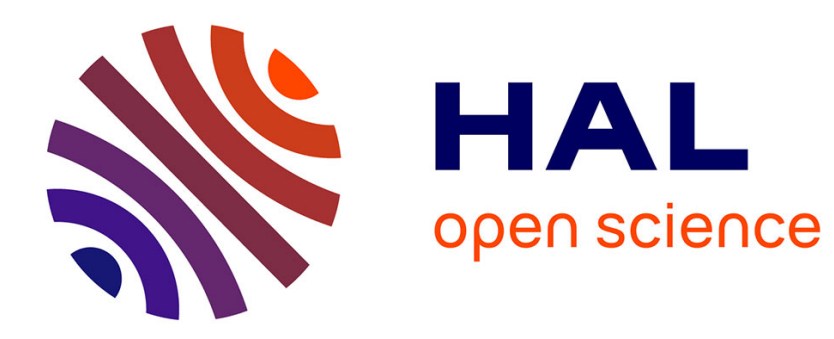

\title{
L'idéologie de l'identité nationale, un facteur de désagrégation de la société
}

\author{
Laurent Bazin
}

\section{To cite this version:}

Laurent Bazin. L'idéologie de l'identité nationale, un facteur de désagrégation de la société: Éclairages à partir de la Côte d'Ivoire. Savoir / Agir , 2007, 2, pp.61-69. hal-00445531

\section{HAL Id: hal-00445531 \\ https://hal.science/hal-00445531}

Submitted on 8 Jan 2010

HAL is a multi-disciplinary open access archive for the deposit and dissemination of scientific research documents, whether they are published or not. The documents may come from teaching and research institutions in France or abroad, or from public or private research centers.
L'archive ouverte pluridisciplinaire HAL, est destinée au dépôt et à la diffusion de documents scientifiques de niveau recherche, publiés ou non, émanant des établissements d'enseignement et de recherche français ou étrangers, des laboratoires publics ou privés. 


\title{
L'idéologie de l'identité nationale, un facteur de désagrégation de la société \\ Éclairages à partir de la Côte d'Ivoire
}

Laurent Bazin

CNRS-CLERSÉ

\author{
Pré-publication d'un article paru dans la revue Savoir/Agir (éd. du Croquant) \\ $\mathrm{n}^{\circ} 2$, 2007, pages 61-69 \\ Sous réserve des modifications éditoriales apportées par la revue
}

\begin{abstract}
Résumé
En Côte d'Ivoire, le terme « ivoirité » était introduit en décembre 1994 dans le débat politique à la faveur de la promulgation d'une loi électorale modifiant les critères d'accès au droit de vote et à l'éligibilité à la présidence de la République. La notion a fonctionné comme un piège idéologique redoutable. Elle est immédiatement venue occuper une place centrale dans le débat politique ivoirien où elle est devenue un opérateur de désagrégation de la société dans un processus conflictuel de plus en plus violent. L'auteur montre ici comment elle a contribué à la radicalisation des conflits qui a mené au déclenchement d'un coup d'État en 1999, puis de la guerre en 2002.
\end{abstract}

\section{Mots clés}

Côte d'Ivoire - Ivoirité - Identité nationale

Laurent Bazin est chargé de recherche au CNRS. Il est membre du Centre Lillois d'Etudes et de Recherches Sociologiques et Économiques (CLERSÉ - UMR 8019). Il collabore à l'axe «Travail et mondialisation » de l'UMR «Développement et sociétés » (IRD, Paris 1). 
En Côte d'Ivoire, le terme "ivoirité » était introduit en décembre 1994 dans le débat politique à la faveur de la promulgation d'une loi électorale modifiant les critères d'accès au droit de vote et à l'éligibilité à la présidence de la République. La notion a fonctionné comme un piège idéologique redoutable. Elle est immédiatement venue occuper une place centrale dans le débat politique ivoirien où elle est devenue un opérateur de désagrégation de la société dans un processus conflictuel de plus en plus violent. Après l'accord de paix encore fragile conclu dans l'année 2007 entre les deux parties belligérantes, les points qui demeurent irrésolus dérivent directement de la mise en application de l'idéologie de l'identité nationale, et notamment ce qu'on désigne localement comme processus d'identification. Il s'agit de vérifier dans toute la population qui peut légitimement relever de la nationalité ivoirienne et qui n'en ressortit pas : une tâche impossible et qui s'avère une source infinie de conflits, de frustrations et d'humiliations individuelles et collectives. À travers la question de l'ivoirité, c'est-àdire l'idée que l'État puise sa légitimité dans l'autochtonie du peuple ivoirien, la production d'une idéologie de l'identité nationale était un phénomène entièrement nouveau en Côte d'Ivoire en 1994. Ce texte s'efforce d'en fournir une compréhension, en explicitant ses enjeux en termes électoraux puis idéologiques et symboliques, dans un double objectif final : montrer comment cette expérience ivoirienne peut aider à comprendre la situation française présente; fournir quelques éléments d'explication de l'apparition dans les années 1990 de phénomènes semblables dans le monde entier et suggérer la fécondité d'une comparaison internationale pour l'analyser.

\section{Un enjeu électoral}

Rappelons tout d'abord dans quelles conditions est apparue la notion d'ivoirité à la fin de l'année 1994'. Le président Félix Houphouët-Boigny venait de mourir un an auparavant et son successeur, le président de l'Assemblée nationale Henri Konan Bédié, assurait la fonction de chef de l'État jusqu'aux élections de 1995. Leader politique de Côte d'Ivoire dès 1945 quand il avait fondé le PDCI (Parti démocratique de Côte d'Ivoire), Houphouët-Boigny avait été président de la République depuis l'indépendance en 1960. Durant trente ans, il avait exercé son pouvoir dans le cadre d'un régime de parti unique, jusqu'à être contraint de légaliser des partis et une presse d'opposition en 1990. Son opposant « historique », Laurent Gbagbo, avait fondé le Front populaire ivoirien (FPI) et s'était présenté comme challenger de l'opposition aux élections présidentielles de 1990, obtenant environ $20 \%$ des suffrages. Le FPI avait alors abondamment accusé le vieux président de s'être maintenu au pouvoir en faisant massivement voter les étrangers africains, très nombreux en Côte d'Ivoire (au milieu des années 1990, environ un quart de la population recensée pour l'ensemble du pays, $40 \%$ pour Abidjan, la métropole économique et politique). Cette même année 1990 était nommé pour la première fois un premier ministre, en la personne d'Alassane Dramane Ouattara, auparavant gouverneur de la Banque centrale des États d'Afrique de l'ouest (BCEAO). Le premier ministre devint fort logiquement la cible des attaques de l'opposition et notamment du FPI qui lui reprochait d'être de nationalité burkinabè. Après la mort d'Houphouët-Boigny, Alassane Ouattara en rivalité ouverte avec Konan

\footnotetext{
${ }^{1}$ Pour des raisons de place, il est impossible de présenter ici la complexité de la situation ivoirienne. Le texte est donc centré uniquement sur les enjeux du conflit autour de l'ivoirité. Pour une analyse plus détaillée se reporter à : Laurent Bazin, 2005 : «Rébellions enchaînées en Côte-d'Ivoire ", Chimères, n $^{\circ}$ 56, pp. 15-34 (entretien réalisé par Monique Selim), http://halshs.archives-ouvertes.fr/halshs-00006510.
} 
Bédié prit la tête d'une scission de l'ex-parti unique et fit fonder par ses partisans un nouveau parti politique : le RDR (Rassemblement des républicains). Le conflit politique entre le pouvoir et l'opposition devint dès lors une compétition tripartite entre les trois principales forces politiques, le PDCI, le FPI et le RDR. Durant l'année 1994, tandis que le PDCI se scindait en deux partis ennemis, le FPI mobilisait contre le vote des étrangers dans une campagne de presse virulente où ces derniers étaient qualifiés de « bétail électoral ».

En décembre 1994, le nouveau code électoral fit l'effet d'un formidable coup de théâtre en raison de deux dispositions : il retirait le droit de vote aux étrangers ressortissants des pays voisins et spécifiait comme condition pour être candidat à l'élection présidentielle qu'il fallait être "né ivoirien», de père et de mère eux-mêmes "nés ivoiriens ». Il opérait un brusque retournement de situation et atteignait simultanément plusieurs objectifs. Il privait le FPI de son principal thème de campagne, l'illégitimité d'un pouvoir reposant sur les suffrages des étrangers, et récupérait ce thème de campagne en en déviant la portée contre Alassane Ouattara et ses partisans. Il introduisait une astuce législative qui permettait d'écarter durablement ce dernier de la compétition électorale. De plus, le nouveau texte de loi permettait à l'héritier d'Houphouët-Boigny - une personnalité sans charisme, contestée, salie par un scandale sur de gigantesques détournements de fonds publics à la fin des années 1960 - d'apparaître comme le défenseur d'une « identité nationale » autochtone contre une menace représentée par les étrangers. Enfin, le thème de l'ivoirité, qui n'avait jamais été évoqué auparavant, a permis d'évacuer du devant de la scène toute autre question, les débats étant désormais centrés sur les questions identitaires et les déchirements qu'elles n'ont pas manqué de susciter, dont la violence est allé croissante.

On comprendra que si les conséquences de ces opérations électorales ont été de grande ampleur, c'est que la question de l'ivoirité n'est pas seulement un gadget idéologique, produit par hasard lors d'une compétition électorale ou tous les (mauvais) coups semblent permis. Elle marque un changement radical dans la conception même de la légitimité de l'État et de son enracinement dans la société. Mais avant d'en proposer une analyse, il est nécessaire d'expliquer pourquoi ces deux dispositions ont constitué en elles-mêmes une pomme de discorde.

\section{Nationaux, étrangers : identification conflictuelle et dénis de citoyenneté}

Tout d'abord, la question désignée dans les débats politiques ivoiriens sous le terme d'identification: une fois privée de son thème de contestation principal (le vote des étrangers), l'opposition se mit à dénoncer le fait que les autorités avaient distribué des cartes d'identité indûment à des étrangers. Elle réclamait donc, pour accepter la tenue d'élections, la vérification de l'authenticité de la nationalité ivoirienne pour tous ceux qui en bénéficiaient. Ce processus, dit d'identification, n'est toujours pas réalisé à la fin 2007. Le règlement de cette question demeure une condition requise par le FPI (qui a pris le pouvoir en 2000) pour l'organisation des premières élections qui devraient avoir lieu depuis le déclenchement de la guerre en 2002. L'identification est une opération éminemment litigieuse, car elle suppose de remettre en question la validité des cartes d'identité et de départager ceux qui pourraient légitimement revendiquer la nationalité ivoirienne de ceux qui l'auraient usurpé. Il s'agit donc de destituer de leur nationalité certains citoyens ou certaines catégories de citoyens. 
La question est d'autant moins facile à résoudre que les processus migratoires liés à la mise en place de l'agriculture d'exportation ont intriqué les populations autochtones des zones forestières du Sud (où se cultive le cacao, café, etc.), les migrants en provenance des autres régions du pays et ceux qui sont originaires des pays voisins : Burkina-Faso, Mali, Guinée... À ces migrations en milieu rural s'ajoutent les migrations urbaines. Or, que ce soit dans les campagnes ou dans les villes, il existe une grande proximité entre les migrants venus du Nord de la Côte d'Ivoire et ceux qui proviennent de ces trois pays voisins : tous sont majoritairement musulmans et fréquemment désignés par l'ethnonyme générique « dioula » ${ }^{2}$. En ce qui concerne les « originaires du Nord» (qui sont en fait présents dans tout le pays), depuis toujours plus ou moins assimilés aux originaires des pays sahéliens, le processus de remise en cause de leur appartenance nationale était en fait amorcé plus tôt. En particulier, l'introduction en 1992 de cartes de séjour obligatoires pour tous les résidents étrangers avait donné lieu, sous prétexte de vérifier la « régularité des situations » (comme en France), à de nombreux harcèlement des musulmans en général, tendanciellement refoulés dans la catégorie d'étrangers et auxquels des pots de vins étaient extorqués par les policiers (ironiquement, si on considère la suite de l'histoire, Alassane Ouattara était alors premier ministre).

En second lieu, la question de la nationalité d'Alassane Ouattara est demeurée un abcès litigieux qui a empoisonné le débat politique et qui a servi de catalyseur aux conflits. Pourquoi ? Depuis les début de la décolonisation, l'État ivoirien était accusé par ses opposants de servir des intérêts étrangers ; les polémiques sur l' " ivoirité » permettaient de rejeter cette accusation sur la seule personne de l'ancien premier ministre avec une fixation d'autant plus forte qu'il était lui-même dénoncé comme étranger et qu'il était soutenu par les États occidentaux. Il est d'autre part la figure politique qui a symbolisé à travers sa personne la représentation ambiguë de l'appartenance à l'ensemble national des régions du Nord, de ceux qui en sont originaires ou des musulmans en général... et de tous ceux susceptibles d'être désignés de la sorte ou de se reconnaître dans cette désignation. Ainsi, la "clause d'ivoirité » qui l'excluait de la compétition électorale était un emblème de l'exclusion ressentie par une grande partie de la population dite du Nord. Un malaise profond était entretenu par les mauvais traitements que subissait l'expremier ministre, dont la nationalité ivoirienne était mise en cause, dont on contestait le droit d'exercer la fonction de chef de l'État au nom d'origines « douteuses ». Était-il ivoirien? Né ivoirien? Était-il burkinabè ? Ou avait-il abandonné la nationalité ivoirienne pour la nationalité burkinabè ? Son père, sa mère habitaient-ils bien dans le Nord de la Côte d'Ivoire ? Étaient-il ivoiriens? Nés ivoiriens ? Le leader du RDR a d'ailleurs lui-même exploité autant qu'il l'a pu cette image de victime, pour figurer comme le défenseur des musulmans et des nordistes dont il espérait les soutiens et les suffrages.

Enfin, c'est toute la question de la place des «étrangers » en Côte d'Ivoire qui était posée sur la place publique, avec des surenchères rhétoriques, des mesures législatives (exclusion du droit à la propriété foncière, exclusion de l'emploi) et des attaques xénophobes qui ont culminé d'abord en 1999 (année où des milliers de planteurs burkinabès dans le sud-ouest furent chassés des terres qu'ils exploitaient; troubles qui ne sont probablement pas étrangers au soutien que le Burkina-Faso a apporté à

\footnotetext{
${ }^{2}$ Dioula signifie « commerçant ». La langue dioula est un dérivé simplifié de la langue bambara parlée au Mali. En raison précisément de son rôle de lingua franca du commerce (et de l'islam), elle est une langue véhiculaire répandue dans toute l'Afrique de l'ouest, et se trouve avec le français (langue officielle) la seconde langue de communication en Côte d'Ivoire.
} 
l'insurrection armée en 2002) puis durant la guerre. Cette question est d'autant plus épineuse que les «étrangers» représentaient un quart de la population au milieu des années 1990 et qu'une grande partie d'entre eux ne sont pas des migrants, mais sont nés en Côte d'Ivoire de familles qui sont installées dans le pays depuis plusieurs générations.

Un des enseignements les plus criants du conflit en Côte d'Ivoire est que la déstabilisation des "étrangers » se répercute par ondes de choc successives sur des fractions entières de la population qu'elle déséquilibre à leur tour. Dans le même mouvement, la promotion de l'identité nationale ne contribue pas à unifier la nation: bien au contraire, en faisant porter un débat sur une norme de l'identité autochtone et sur la plus ou moins grande conformité à cette norme d'individus ou de catégories particulières d'individus, elle entraîne une suspicion généralisée et des remises en cause potentiellement infinies de la «vraie» identité de chacun, débouchant sur une fragmentation du corps social et sur des antagonismes.

\section{Le passage à une citoyenneté définie par l'autochtonie}

Il convient maintenant d'examiner ce qui se joue dans la cristallisation des conflits sur la question des identités. Le processus qui est en cours et qui amplifie les tensions politiques, c'est la production d'une «identité nationale » autochtone : l'« ivoirité ». Bien entendu, beaucoup d'autres facteurs économiques et sociaux, enchevêtrés, sont à l'origine de la radicalisation des tensions politiques et du déclenchement du conflit armé. Il serait trop long de les détailler dans cet article. En revanche, ce qui est intéressant de souligner dans le cadre d'une réflexion sur la notion d' «identité nationale ", c'est qu'une transformation radicale s'opère à travers ces polémiques houleuses sur l'« ivoirité » qu'emblématise la mise en cause de la nationalité et des origines d'Alassane Ouattara. C'est l'ancrage symbolique de l'État qui est ainsi redéfini de manière violente.

L'inversion qui se produit au début des années 1990 est particulièrement spectaculaire en Côte d'Ivoire. Depuis la décolonisation, l'État avait assumé une connivence avec des intérêts étrangers à différents niveaux. Le régime d'Houphouët-Boigny se faisait une gloire d'être le plus fidèle allié en Afrique des puissances occidentales (France, ÉtatsUnis) en termes géopolitiques et économiques. Le développement du pays, dès la période de «mise en valeur» de la colonie au début du $\mathrm{XX}^{\mathrm{e}}$ siècle, avait reposé sur des capitaux occidentaux et sur la main-d'œuvre constituée par les migrants du nord et des pays sahéliens. En dépit même de mesures d'ivoirisation qui avait été mises en place en 1974 et qui réservaient théoriquement les emplois «formels» aux nationaux, la politique en la matière reposait sur la plus grande liberté de circulation et d'installation des migrants, qu'ils soient originaires de la Côte d'Ivoire ou de pays étrangers. Comme Jean-Pierre Dozon l'a très bien formulé, l'État figurait comme un État allochtone ${ }^{3}$.

La population entière de Côte-d'Ivoire était d'ailleurs supposée être venue des pays limitrophes à une époque relativement récente. Les grands groupes ethniques qui composaient le pays étaient donc tous allochtones : les Akans seraient venus du Ghana, les Malinkés du Mali, les Sénoufos du Burkina-Faso, les Krous du Libéria, etc. Cette représentation d'un peuplement de la nation à partir de l'extérieur était l'histoire officielle enseignée dans les écoles.

\footnotetext{
3 Jean-Pierre Dozon : «L'étranger et l'allochtone en Côte-d'Ivoire », in B. Contamin et H. Memel-Fotê (dir.), Le modèle ivoirien en questions, Karthala-ORSTOM, 1997 : 779-798.
} 
Du point de vue idéologique et politique, «l'ivoirité » des années 1990 est un tout autre phénomène que l'ivoirisation des années 1970. En dépit d'accès de xénophobie récurrents et d'une discrimination selon la nationalité inscrite dans le droit (du travail), la nationalité était demeurée en Côte d'Ivoire une catégorie qui avait relativement peu de sens et d'importance. Jusqu'à la promulgation du code électoral de 1994, les étrangers ressortissant des États de la CEDEAO ${ }^{4}$ avaient le droit de vote, et il n'était pas rare que le gouvernement compte parmi ses membres des ministres non ivoiriens ${ }^{5}$. Même s'il faudrait nuancer le propos, peu de demandes d'acquisition de la nationalité ivoirienne étaient enregistrées de la part des nombreux «étrangers » recensés, souvent présents en Côte d'Ivoire depuis plusieurs générations : l'installation des non-nationaux et leur "participation au développement économique» étaient libres (avec une restriction pour l'accès aux emplois « formels »). En outre, l'accès au droit de vote n'était pas un enjeu crucial dans le cadre d'un régime de parti unique.

Avec «l'ivoirité", tout a changé brusquement puisque cette notion intervenait précisément pour signifier que l' «autochtonie ivoirienne » devenait le seul socle de la légitimité politique : il fallait désormais "être né ivoirien, de père et de mère euxmêmes nés ivoiriens » pour prétendre représenter le pays.

En fait, si la nationalité avait peu d'importance auparavant, c'est que le socle idéologique sur lequel s'était bâti l'État postcolonial après 1960 ainsi que l'idée même de citoyenneté étaient en réalité fondés sur la participation au développement économique et à la modernisation de la société. Cette conception de la citoyenneté a fonctionné jusque dans les années 1980. Elle a été balayée par la récession économique et surtout invalidée par la mise en place des plans d'ajustement structurel qui ont déstabilisé l'État et dont l'inspiration était, à l'origine, celle d'un néolibéralisme radical.

\section{En conclusion : les effets boomerang de l'État importét}

Ce n'est pas la vague néolibérale des années 1980 qui a implanté un identitarisme d'État en lieu et place des idéaux du développement qu'elle détruisait. Mais elle a provoqué une disqualification de l'État, d'autant plus inexorable que la situation économique n'a cessé de se dégrader. Elle a donc sapé le mode de légitimation de l'État, sans fournir en elle-même d'idéologie de remplacement sur laquelle les gouvernants pouvaient s'appuyer : un État quel qu'il soit ne peut guère trouver matière à légitimer son autorité dans les principes du marché, supposés bénéfiques, d'autant moins quand ils sont imposés de l'extérieur. C'est donc dans le vide idéologique ainsi constitué, au secours d'un président sans crédit, que s'est glissée la production d'une idéologie de l'autochtonie, impensable auparavant. Une fois enclenché, le processus d'autochtonisation de l'État a déroulé ses effets d'une manière extrêmement rapide. Il a accentué les logiques de fragmentation qui ont largement contribué au déclenchement du conflit armé en 2002.

Dans la mesure où l'on prend conscience de la généralité du phénomène, l'évolution tragique de la Côte d'Ivoire n'apparaît plus comme un conflit singulier et exotique, qui

\footnotetext{
${ }^{4}$ Communauté Économique des États d'Afrique de l'Ouest.

${ }^{5}$ Roch Yao Gnabéli, 2002 : «Luttes politiques et reconstruction des identités collectives en Côte d'Ivoire (1990-2001) », Journal des anthropologues, 88-89 : 161-184.

${ }^{6}$ Un clin d'œil à Bertrand Badie : L'État importé, Fayard, 1992. Si la formule est très juste, inversement les pays du Tiers-monde, parce que leur position est plus précaire, sont dans bien des aspects les laboratoires de transformations qui sont ensuite implantées dans les pays dits développés.
} 
resterait incompréhensible à ceux qui ne sont pas familiers du pays. Avec son histoire particulière, la Côte d'Ivoire est dans les années 1990 le théâtre de processus analogues à ce qui s'est passé dans beaucoup d'autres pays. Par exemple la disparition du socialisme en ex-Yougoslavie a donné lieu à l'instauration d'États identitaires à travers un processus violent d' " épuration ethnique ». Dans les États de l'ex-URSS, l'idéologie de l'identité nationale s'est très rapidement substituée à l'idéologie communiste, y compris lorsque le régime autoritaire postsoviétique a bloqué l'introduction de l'économie de marché comme cela a été le cas en Ouzbékistan ${ }^{7}$. En Amérique latine, et singulièrement en Bolivie où le choc néolibéral a été extrême et où en 2005 un "président indien » a été élu, la reconceptualisation d'une «identité de la nation» incorpore la figure des populations autochtones, les Amérindiens. Ce changement de l'assise de l'État s'opère dans un contexte international marqué par l'institutionnalisation, par l'ONU, des peuples autochtones. Dans l'ensemble de ces situations nationales (on pourrait en citer bien d'autres) les processus sont analogues et contemporains de l'implantation de l'ivoirité en Côte d'Ivoire. La situation de la France en 2007 peut également être rapportée à ce phénomène généralisé. Sous l'impulsion de Nicolas Sarkozy et de ses conseillers les plus proches, le gouvernement a délibérément emprunté à l'extrême droite la thématique de la promotion et de la sauvegarde d'une « identité nationale » qui serait menacée par les immigrants étrangers. Ce faisant, il ne s'aperçoit même pas qu'il importe très directement une forme de légitimation de l'État dont les prémices sont nés sur les débris de l'héritage stalinien... ou sur les ruines du développement en Afrique. À moins que... Nicolas Sarkozy ne déclarait-il pas à Dakar que l'Afrique était restée en dehors de l'histoire? Un signe flagrant d'inculture ou le dessein de brouiller les pistes?

\footnotetext{
${ }^{7}$ Laurent Bazin, 2007 : «Construction nationale, travail et discipline en Ouzbékistan », in Hernandez, Ould-Ahmed, Papail \& Phélinas (dir): L'action collective à l'épreuve de la globalisation. Paris, L'Harmattan : 235-247 ; http://halshs.archives-ouvertes.fr/halshs-00159726 ; Monique Selim : "Travail, idéologie et réflexivité dans le cadre de la globalisation. Perspectives comparatives ", in ibidem : 205234.
} 